\title{
Spacecraft potential effects on electron moments derived from a perfect plasma detector
}

\author{
V. Génot ${ }^{1, *}$ and S. J. Schwartz ${ }^{1}$ \\ ${ }^{1}$ Astronomy Unit, Queen Mary, University of London, Mile End Road, London E1 4NS, England, UK \\ *Now permanently at CESR, Toulouse, France
}

Received: 16 April 2003 - Revised: 14 January 2004 - Accepted: 28 January 2004 - Published: 14 June 2004

\begin{abstract}
A complete computation of the effect of the spacecraft potential on electron moments is presented. We adopt the perfect detector concept to estimate how measured density, velocity and temperature are affected by the constraints imposed by the detector, such as the finite lower energy cutoff and the spacecraft potential. We investigate the role of the potential in different plasma regimes usually crossed by satellites. It appears that the solar wind is the region where the moments are most compromised, as the particle temperature is low. To a lesser extent the moments calculated in the magnetosheath may also deviate from the real moments, displaying up to $40 \%$ overestimation for the density under typical detector operation. The analysis allows us to identify a range of spacecraft potential values which minimizes the variation in the estimation; it is found that it corresponds to the common value adopted by potential controlling experiments.
\end{abstract}

Key words. Space plasma physics (spacecraft sheaths, wakes, charging; experimental and mathematical techniques; instruments and techniques)

\section{Introduction}

The knowledge of plasma parameters, such as density and temperature, which are moments of the particle distribution functions, is fundamental to any analysis both in space and in the laboratory contexts (see the review in Paschmann et al., 1998). In this aim, the spectrometer technique is based on the direct measurement of particles whose trajectories and energies may be modified by the spacecraft electrical potential. Once the effect of the latter is understood, it is possible to extract accurate density, velocity and temperature from

Correspondence to: V. Génot

(vincent.genot@cesr.fr) the spectrometer measurements. If the full set of spectrometer data are available, the moment sums may be performed on the ground after allowing the spacecraft-induced effects. However, limited telemetry often results in limited transmitted particle data, and moments are often computed onboard. Deducing accurate information from such onboard moments requires techniques such as those we develop in this paper.

Whereas the direct detection of the plasma populations with an energy spectrometer seems the most obvious solution, several other alternative methods have been designed to avoid the spectrometer limitations and constraints. These techniques are based either on electrical or wave measurements: the fluctuations in the Langmuir probe collected current result from fluctuations in the density, the temperature and the floating spacecraft potential (Hilgers et al., 1992); differential potential measurements with double probes provide a diagnostic of the density linking it directly to the spacecraft potential (Laakso et al., 1998); finally, the plasma resonance sounder technique offers an accurate measure of the plasma frequency from which density can be deduced (Décréau et al., 1997) (further data analysis may also provide the temperature using thermal noise spectroscopy; MeyerVernet et al., 1998). The range of validity of these methods, as well as their time resolution, differs, but they all either rely on or are un-affected by the spacecraft potential.

In this paper we revisit the concept of the "perfect" plasma detector introduced by Song et al. (1997) and show explicitly the effect of the spacecraft potential. A perfect plasma detector would be an ideal spectrometer, therefore impossible to build, for which no calibration problems (due to age, signalto-noise ratio, poor count statistics, etc.) intervene. The uncertainties in the calculated moments are then purely caused by the technical constraints (lower and upper cutoffs) and the plasma environment which induces the formation of secondary and photoelectrons from the spacecraft. These electrons may in turn come back to the detector and pollute the measure; we shall not discuss this effect here (see, for instance, Szita et al., 2001). This electron emission generates 
a deficit of negative charge on the spacecraft which will then generally charge positively.

This potential is of course also the cause of uncertainties, as low energy plasma electrons characteristics will be modified as they approach the detector. In this article, we adopt a simple approach to the way the potential affects electrons. It is known as the scalar correction and assumes that the particle trajectories are purely radial to the detector. This is a crude assumption and some authors have developed strategies to overcome it (Scime et al., 1994; Bouhram et al., 2002).

However, an analytical approach to the correct modification of particle trajectories is difficult, even in the case of a 3-D purely spherical detector. Moreover, the spacecraft geometry and fabric also have to be considered if one wants to conduct a detailed study. At this stage an analytical path is no longer the best choice. Global Particle In Cell (PIC) simulations may provide the solution as they can take into account geometry and fabric, in addition to computing individual particle trajectories (see, for instance, Singh et al., 2001, in the Polar context). This has also been studied by the SPINE consortium with the PicUp3D code (Forest et al., 2001; see also http://spis.onecert.fr/picup3d/index.html).

However, on a much simpler scale, the "perfect" plasma detector may provide estimates of relations between the true moments and those calculated, for example, onboard, without compensation for the detector and spacecraft environment. The analytical approach facilitates a survey of the parameter space to analyze the effect on moments. Here we shall investigate the effects of detector energy cutoffs (the lower one is set to avoid the low energy plasma, usually contaminated by photoelectrons), as well as of the spacecraft potential. The potential effect is indeed important but has never been tackled before in this context. In Song et al. (1997), the authors correctly state that in the presence of the spacecraft potential the cutoff energies should be evaluated as the detector's cutoff energies plus (minus) the potential for ions (electrons), but in fact the calculations are done for a null potential and the results are displayed accordingly. In the present paper, we keep the spacecraft potential as a variable throughout the calculations. We finally end up with expressions of the measured moments as functions of the "real" (free space) moments. The expression are solved analytically and the ratios of measured to "real" moments are displayed as a function of the potential. It is therefore possible, for given measured plasma conditions, to estimate the influence of the spacecraft potential. The method resorts to numerical computations only at the last stage; it does, however, require the inversion of a nonlinear integral system. The algorithm proved to be convergent for parameters corresponding to nominal operations (solar wind, magnetosheath and magnetosphere conditions, spacecraft potential up to $\sim 30 \mathrm{~V}$, and lower cutoff $\sim 10 \mathrm{eV}$ ). In a degenerate case (considering a non-drifting Maxwellian function for the velocity distribution of electron population, but retaining the potential), Salem et al. (2001), also in the "perfect" detector frame, ingeniously skipped the demanding inversion task by using a fitting method in restricted density and temperature ranges. This was possible because the measured temperature depended only on the "real" temperature in their case. This method is not applicable for our more general approach.

In the next section we derive the basic equations which link the measured and "real" moments, then in Sect. 3 we briefly discuss the numerical method we developed. In Sect. 4 we present and discuss the results for different typical plasma conditions. Finally, in the conclusion we summarize our results, emphasize their limitations and the possible improvements, and we discuss them in the light of more powerful, but more demanding methods.

\section{Equations of the moments}

In the spacecraft frame the moments of the particle velocity distribution function $f_{s c}$ are given by the following formula $\left(v_{l}, v_{u}\right.$ are the lower and upper speed cutoffs of the detector):

$M(\xi)=\int_{v_{l}}^{v_{u}} \int_{0}^{\pi} \int_{0}^{2 \pi} v_{s c}^{2} d v_{s c} \sin \theta_{s c} d \theta_{s c} d \phi_{s c} \xi f_{s c}\left(v_{s c}, \theta_{s c}, \phi_{s c}\right),(1)$

$\xi$ is a parameter which may be a scalar, a vector or a tensor (or higher order) and $M$ is then a moment of order one, two or three, respectively. The physical quantities derived from the measured moments values are the density $N$, the velocity vector $\boldsymbol{V}$ and the pressure tensor $\bar{P}$ given by:

$N=M(1)$

$N \boldsymbol{V}=M\left(\boldsymbol{v}_{s c}\right)$

$\bar{P}=m M\left(\boldsymbol{v}_{s c} \boldsymbol{v}_{s c}\right)-m N \boldsymbol{V} \boldsymbol{V}$,

where $m$ is the mass of the particle. Note that some physical quantities of interest (e.g. $\bar{P})$ involve combinations of the basic moments $M(\xi)$.

In the following we shall consider an electron detector; the charge and mass of the electron are $-e$ and $m_{e}$, respectively. If $\Phi_{s c}$ denotes the spacecraft potential, the conservation of energy of an electron can be written:

$v_{s c}^{2}=v^{2}-\mathcal{E}$,

where $v$ is the velocity in "free space" and $\mathcal{E}$ is defined by

$\mathcal{E}=-\frac{2 e \Phi_{s c}}{m_{e}}$.

It corresponds to the free space energy of an electron which would have zero energy in the spacecraft frame; it is a negative quantity for most of the plasma conditions encountered in space. Along a trajectory in phase space the distribution function remains constant (Liouville theorem):

$f_{s c}\left(v_{s c}, \theta_{s c}, \phi_{s c}\right)=f(v, \theta, \phi)$.

Far from the spacecraft, in "free space" (respective quantities are without subscripts), we assume a thermal equilibrium such that the distribution function $f$ may be assumed to 
be a simple Maxwellian drifting with the velocity $\boldsymbol{V}_{0}$ in the spacecraft frame and of thermal temperature $T_{0}$. Integrating this function over $4 \pi$ steradian and over all velocities (which corresponds to an absence of cutoffs, or $v_{l}=0$ and $v_{u}=\infty$ ) gives the total plasma density, $N_{0} . f$ is given by:

$$
f(v, \theta, \phi)=N_{0}\left(\frac{m_{e}}{2 \pi k T_{0}}\right)^{3 / 2} \exp \left(-\frac{m_{e}}{2 k T_{0}}\left(\boldsymbol{v}-\boldsymbol{V}_{0}\right)^{2}\right)
$$

or

$$
\begin{aligned}
& f(v, \theta, \phi)=N_{0}\left(\frac{m_{e}}{2 \pi k T_{0}}\right)^{3 / 2} \exp \\
&\left(\frac{m_{e}}{2 k T_{0}}\left(-v^{2}-V_{0}^{2}+2 v V_{0} \cos \theta\right)\right) .
\end{aligned}
$$

Here we have defined the $z$ direction to correspond to that of the drift velocity $\boldsymbol{V}_{0}$. The more general case, for example, in which the magnetic field defines a second direction associated with an anisotropy in temperature, is considerably more difficult to treat analytically. We now consider that the effect of the spacecraft potential is only effective in the radial direction. This so-called scalar approximation can be expressed by $\theta_{s c}=\theta$ and $\phi_{s c}=\phi$. Only the velocity magnitude is affected (Eq. 5). We can then rewrite Eq. (1) with respect to the variables $(v, \theta, \phi)$. The integration element $d v_{s c}$ has to be changed according to $v_{s c} d v_{s c}=v d v$. After some algebra, the measured density, velocity and diagonal elements of the pressure tensor can be expressed by:

$$
\begin{aligned}
& N=\left(\frac{m_{e}}{2 \pi k T_{0}}\right)^{1 / 2} \frac{N_{0}}{V_{0}} \int_{v_{L}}^{v_{U}} d v \\
& \quad \sqrt{v^{2}-\mathcal{E}}\left(e^{-\frac{m_{e}}{2 k T_{0}}\left(v-V_{0}\right)^{2}}-e^{-\frac{m_{e}}{2 k T_{0}}\left(v+V_{0}\right)^{2}}\right) \\
& N V_{x}=N V_{y}=0
\end{aligned}
$$

$$
\begin{gathered}
N V_{z}=\left(\frac{m_{e}}{2 \pi k T_{0}}\right)^{1 / 2} \frac{N_{0}}{V_{0}} \int_{v_{L}}^{v_{U}} d v \\
\quad\left[\left(v^{2}-\mathcal{E}\right)\left(e^{-\frac{m_{e}}{2 k T_{0}}\left(v-V_{0}\right)^{2}}+e^{-\frac{m_{e}}{2 k T_{0}}\left(v+V_{0}\right)^{2}}\right)\right. \\
\left.-\frac{v^{2}-\mathcal{E}}{v} \frac{k T_{0}}{m_{e} V_{0}}\left(e^{-\frac{m_{e}}{2 k T_{0}}\left(v-V_{0}\right)^{2}}-e^{-\frac{m_{e}}{2 k T_{0}}\left(v+V_{0}\right)^{2}}\right)\right] \\
P_{x}=P_{y}=\frac{N_{0}}{V_{0}^{2}}\left(\frac{m_{e} k T_{0}}{2 \pi}\right)^{1 / 2} \int_{v_{L}}^{v_{U}} d v \frac{\left(v^{2}-\mathcal{E}\right)^{\frac{3}{2}}}{v} \\
\quad\left[e^{-\frac{m_{e}}{2 k T_{0}}\left(v-V_{0}\right)^{2}}+e^{-\frac{m_{e}}{2 k T_{0}}\left(v+V_{0}\right)^{2}}\right. \\
\left.-\frac{k T_{0}}{m_{e} v V_{0}}\left(e^{-\frac{m_{e}}{2 k T_{0}}\left(v-V_{0}\right)^{2}}-e^{-\frac{m_{e}}{2 k T_{0}}\left(v+V_{0}\right)^{2}}\right)\right]
\end{gathered}
$$

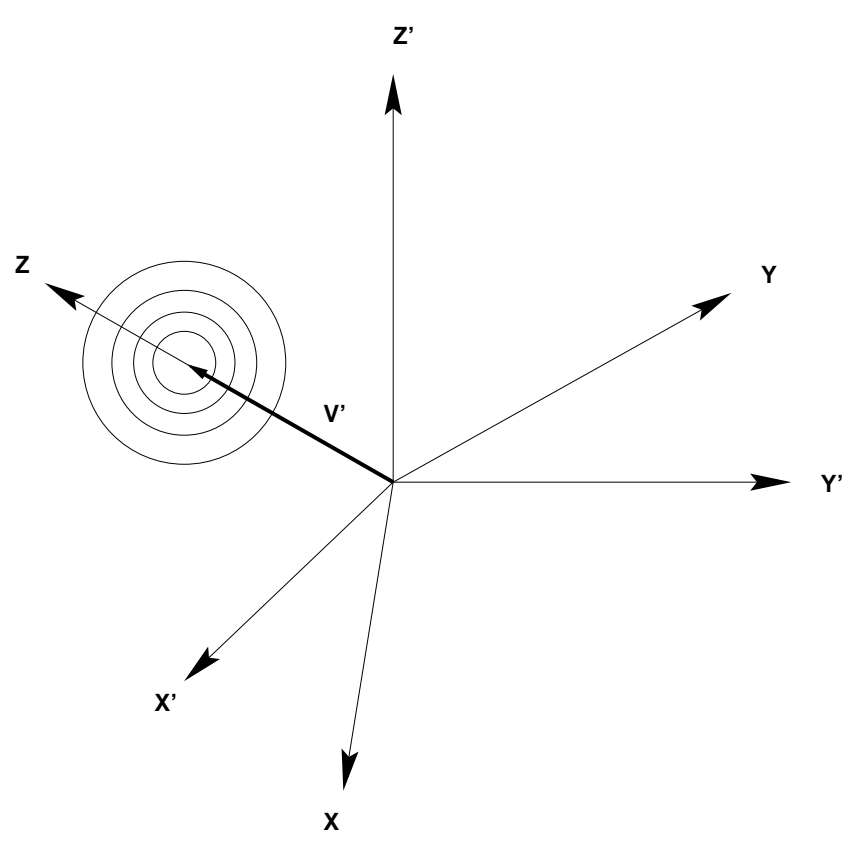

Fig. 1. Sketch of the measurement (or spacecraft) frame $\left(X^{\prime}, Y^{\prime}, Z^{\prime}\right)$ and computation frame $(X, Y, Z)$. The Maxwellian drift velocity is $V^{\prime}$.

$$
\begin{aligned}
P_{z}= & -m_{e} N V_{z}^{2}+\frac{N_{0} m_{e}}{V_{0}}\left(\frac{m_{e}}{2 \pi k T_{0}}\right)^{1 / 2} \int_{v_{L}}^{v_{U}} d v \\
& \left(v^{2}-\mathcal{E}\right)^{\frac{3}{2}}\left[e^{-\frac{m_{e}}{2 k T_{0}}\left(v-V_{0}\right)^{2}}-e^{-\frac{m_{e}}{2 k T_{0}}\left(v+V_{0}\right)^{2}}\right. \\
& -\frac{2 k T_{0}}{m_{e} v V_{0}}\left(e^{-\frac{m_{e}}{2 k T_{0}}\left(v-V_{0}\right)^{2}}+e^{-\frac{m_{e}}{2 k T_{0}}\left(v+V_{0}\right)^{2}}\right) \\
& \left.+2\left(\frac{k T_{0}}{m_{e} v V_{0}}\right)^{2}\left(e^{-\frac{m_{e}}{2 k T_{0}}\left(v-V_{0}\right)^{2}}-e^{-\frac{m_{e}}{2 k T_{0}}\left(v+V_{0}\right)^{2}}\right)\right]
\end{aligned}
$$

with $v_{L, U}=\sqrt{v_{l, u}^{2}+\mathcal{E}}$. The expressions calculated by Song et al. (1997) can be recovered for $\mathcal{E}=0$ and $v_{u}=\infty$; those of Salem et al. (2001) for $V_{0}=0$ and $v_{u}=\infty$.

Let $\mathcal{R}$ be a rotation which transforms the spacecraft measurement frame to a frame where the $z$ axis is aligned with the measured velocity $V^{\prime}$ (see Fig. 1). In the first frame the moments are $N, V^{\prime}=\left(V_{x}^{\prime}, V_{y}^{\prime}, V_{z}^{\prime}\right)$ and $\bar{P}^{\prime}$, whereas in the second frame they are $N$ (scalar quantity), $\boldsymbol{V}=\left(0,0,\left|\boldsymbol{V}^{\prime}\right|\right)$ and $\bar{P}$. As $\mathcal{R}$ conserves the trace of tensors, the temperature $T$ computed from $\bar{P}$ or $\bar{P}^{\prime}$ is the same: $3 N k T=\operatorname{tr}(\bar{P})=\operatorname{tr}\left(\bar{P}^{\prime}\right)$ (tr stands for the trace of the tensor). In the following we shall express the diagonal elements of the pressure tensor in the second frame, and then compute the temperature $T$, frame independent. In practice we do not need to know the details of $\mathcal{R}$ : from the measured velocity $\boldsymbol{V}^{\prime}$, we directly deduce $\boldsymbol{V}$ which is used in the computation. Since the direction of the velocity is unaffected by the potential and speed cutoffs due to the scalar approximation (see Eqs. 11 and 12), the 

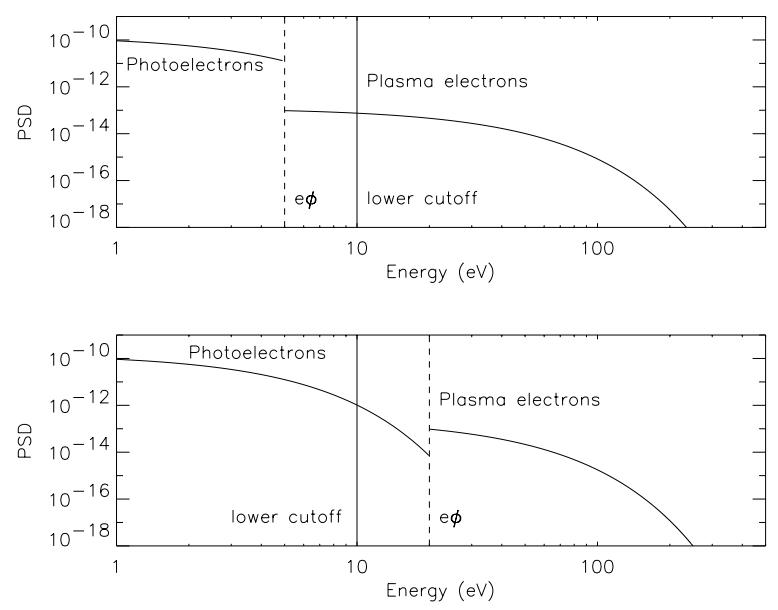

Fig. 2. Electron and photoelectrons distributions for a spacecraft potential smaller than the lower cutoff (upper panel), and for the reverse situation (bottom panel). The portion of the distribution measured by the detector lies to the right of the solid vertical line.

resulting "real" velocity $\boldsymbol{V}_{0}$ can then be expressed back in the original frame as $V_{0} V^{\prime} / V$. This transformation enabled us to perform the integration over angles $\theta$ and $\phi$ analytically. The remaining integrations over the speed $v$ presented in Eqs. (10)-(14) require a computational approach.

From the definition of $v_{L}$, we see that the situation $\sqrt{-\mathcal{E}}>v_{l}$, i.e. when the spacecraft potential reaches values higher than the low energy cutoff, is undefined. Actually, in this case we set $v_{L}=0$ for the computation. In the velocity range $\left[v_{l}, \sqrt{-\mathcal{E}}\right]$, the detector would measure photoelectrons, or more generally secondary electrons, which are emitted by the spacecraft (see Fig. 2). These particles return to the detector as their energy is usually less than $|\mathcal{E}|$. Therefore, we could fill the range $\left[v_{l}, \sqrt{-\mathcal{E}}\right]$ with a model of secondary electron distributions. A popular one considers the superposition of two Maxwellians with thermal energies $\sim 2 \mathrm{eV}$ and $\sim 7 \mathrm{eV}$ (see Grard, 1973, for instance). However, this procedure would add more parameters to our computation and we prefer to restrain our study to the cases $\sqrt{-\mathcal{E}}<v_{l}$. For $m_{e} v_{l}^{2} / 2=10 \mathrm{eV}$, this corresponds to usual solar wind and magnetosheath conditions. Nevertheless, we present a restrictive method (assuming no secondary electrons) for the case $\sqrt{-\mathcal{E}}>v_{l}$ which then also applies to common magnetospheric conditions.

\section{Numerical method}

The interest of the above analysis is to cast estimations based on measured moments to an almost fully analytical form. However, integrations over speeds cannot be determined analytically. The cutoffs are fixed by the particle instrument (actually the upper one does not matter much as even low energy detectors operate up to $\sim 1 \mathrm{keV}$, a value typically $\gg k T$ for which the integrand in Eqs. 10 to 14 tends to zero). Given the triplet $\left(N, V_{z}, T\right)$, the measured values, and the spacecraft
Table 1. Electron parameters used in the simulations.

\begin{tabular}{cccc}
\hline location & density $\left(\mathrm{cm}^{-3}\right)$ & velocity $(\mathrm{km} / \mathrm{s})$ & temperature $(\mathrm{eV})$ \\
\hline solar wind & 5 & 1000 & 10 \\
magnetosheath & 50 & 500 & 20 \\
magnetosphere & 10 & 100 & 100 \\
\hline
\end{tabular}

potential $\Phi_{s c}$, the goal is to invert the following nonlinear set of equations to obtain the corrected triplet $\left(N_{0}, V_{0}, T_{0}\right)$ which defines the "ideal" Maxwellian, free from any spacecraft effects:

$\left\{\begin{array}{l}g_{1}^{\left(v_{l}, v_{u}, \Phi_{s c}\right)}\left(N_{0}, V_{0}, T_{0}\right)-N=0 \\ g_{2}^{\left(v_{l}, v_{u}, \Phi_{s c}\right)}\left(N_{0}, V_{0}, T_{0}\right)-N V_{z}=0 \\ g_{3}^{\left(v_{l}, v_{u}, \Phi_{s c}\right)}\left(N_{0}, V_{0}, T_{0}\right)-3 N k T=0,\end{array}\right.$

$g_{1}$ is the RHS term of Eq. (10), $g_{2}$ is the RHS term of Eq. (12) and $g_{3}$ is twice the RHS term of Eq. (13) added to the RHS term of Eq. (14) minus $m_{e} N V_{z}^{2}$. In practice, $N, V_{z}, T, \Phi_{s c}$ are functions of time and the system above has to be inverted for each data record. Recall that the direction of the velocity $V_{0}$ is the same as the measured $V^{\prime}$.

The integration is tackled by a robust 100-point Gaussian quadrature routine, whereas the nonlinear system solver is based on a Newton-Raphson algorithm (Press et al., 1992). We tested the convergence by setting $\left(v_{l}, v_{u}, \Phi_{s c}\right)=(0, \infty, 0)$ : as expected, we recovered the measured values to the required precision. Finally, to validate the method, we checked that for $\Phi_{s c}=0$ our results agreed with Song et al. (1997).

\section{Results and discussion}

Three different cases are presented in the following study, each corresponding to plasma conditions in the solar wind, the magnetosheath and the magnetosphere, respectively (see Table 1). We set the measured values of the moments and the lower/upper cutoffs of the detector. The lower cutoff is set to $10 \mathrm{eV}$, unless it is otherwise stated; this is consistent with values generally used on electron analyzers aboard existing satellites, like the Wind 3DP experiment (Lin et al., 1995) or the Cluster PEACE experiment (Johnstone et al., 1997). The upper cutoff is set to $1 \mathrm{keV}$. The range of potential variations starts at $-2 \mathrm{~V}$ to show the continuity of the numerical method, although negative values are rarely encountered. It extends up to the lower energy cutoff.

For each given set of measured moment and potential values, the method is run and returns the "perfect" moments which should be measured with a "perfect" detector. Then we compute the ratios $r_{N}=\frac{N-N_{0}}{N_{0}}, r_{v}=\frac{V_{z}-V_{0}}{V_{0}}$, $r_{T}=\frac{T-T_{0}}{T_{0}}$ which are displayed in Figs. 3, 4, and 5. Values smaller (larger) than zero stand for an under- (over-) estimation. We also plot the value $I_{e}=\left(r_{N}^{2}+r_{v}^{2}+r_{T}^{2}\right)^{1 / 2}$ which 


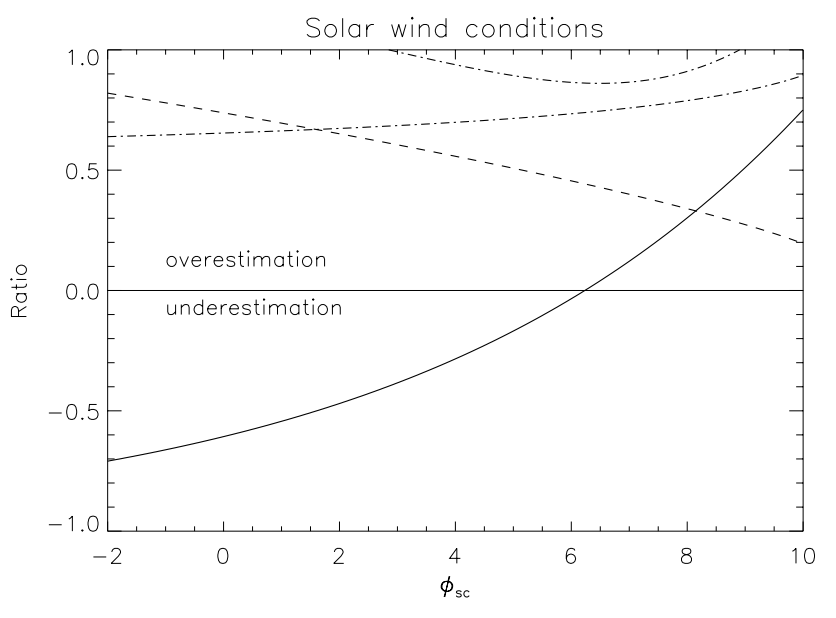

Fig. 3. Estimation ratios (relative differences between measured and corrected moments) in the solar wind case: plasma density ratio $r_{N}$ (solid line), bulk speed ratio $r_{v}$ (dashed line), temperature ratio $r_{T}$ (dot-dashed line). The upper curve is the estimation index $I_{e}$.

globally estimates the degree of deviation from the real moments.

From Figs. 3, 4, and 5 the general behaviour of the ratios above are the following:

- for small values of the spacecraft potential, the density is underestimated, but as the potential increases it becomes overestimated,

- for increasing potential, the overestimation of the velocity magnitude decreases,

- and for increasing potential, the overestimation of the temperature slightly increases.

These general trends do not depend on the specific plasma conditions, although these conditions monitor the amplitudes of the respective variations. The first point noted above is straightforward: if the detector misses a part of the distribution function, one expects to obtain a diminished density. However, as the potential increases, the distribution is shifted to higher energies where the 3-D velocity space volume is larger (or equivalently, the $\sqrt{v^{2}-\mathcal{E}}$ weighting factor which survives in Eq. 10 after the angular integrations is larger). The ratio $r_{N}$ goes through the null value which indicates that there exists a given value of the spacecraft potential for which the correct density is measured by the detector. This was also noticed by Salem et al. (2001). For this critical value of the potential $\left(\Phi_{\text {crit }}\right)$ a sufficient portion of the under-sampled distribution is shifted towards high energies, in such a way that each value of the distribution function contributes more than it should to the integral resulting in the density (see below for a discussion of the variations of $\left.\Phi_{\text {crit }}\right)$. No such critical value exists for the velocity and temperature (at least for typical plasma parameters). For solar wind conditions the range of values corresponding to under/overestimation can be quite large: from $60 \%$ underestimation for zero potential it goes up to $75 \%$ overestimation in

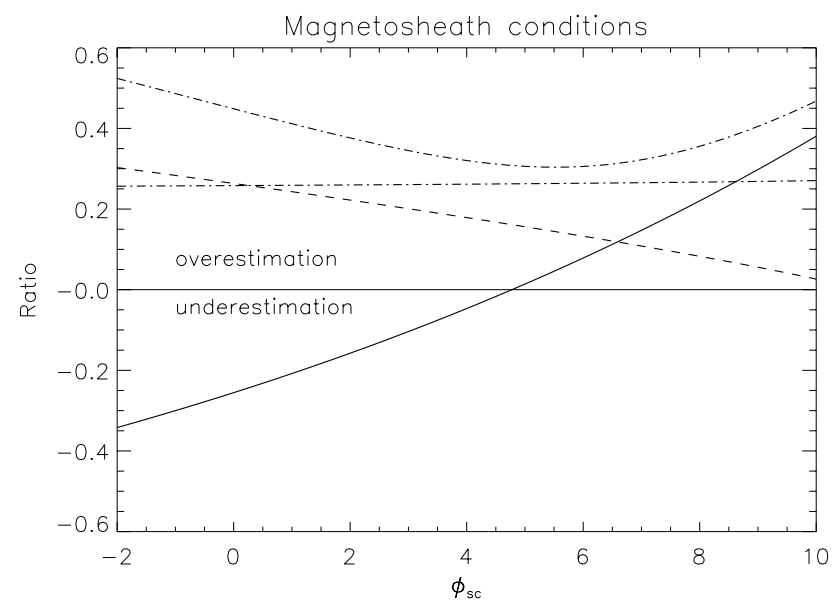

Fig. 4. Estimation ratios as in Fig. 3 but for the magnetosheath case: $r_{N}$ (solid line), $r_{v}$ (dashed line), $r_{T}$ (dot-dashed line). The upper curve is the estimation index $I_{e}$.

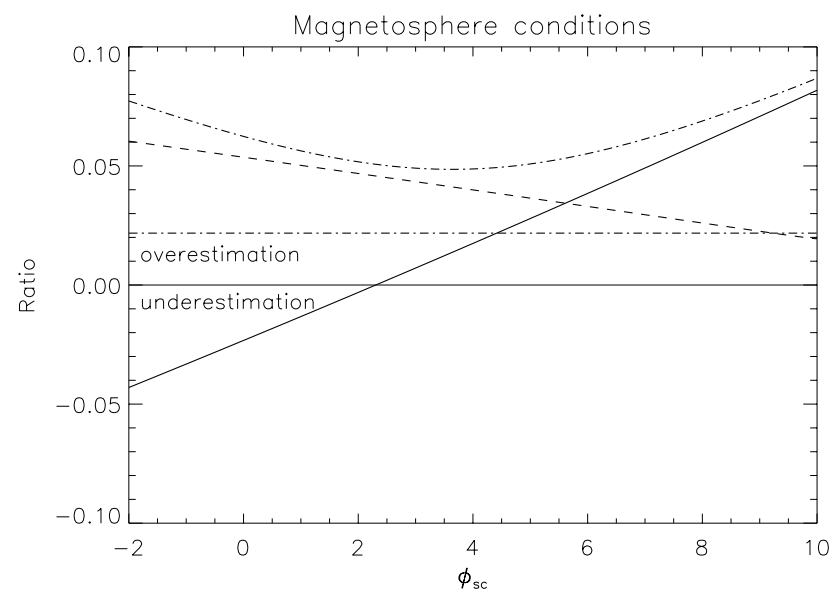

Fig. 5. Estimation ratios as in Fig. 3 but for the magnetosphere case: $r_{N}$ (solid line), $r_{v}$ (dashed line), $r_{T}$ (dot-dashed line). The upper curve is the estimation index $I_{e}$.

the case where the spacecraft potential and the lower energy cutoff are equal. The range spanned by the velocity and temperature is much smaller; however, the velocity measure can reach a $75 \%$ overestimation for zero potential.

As one might expect the width of the distribution function, which is affected by the potential, is a key parameter. Indeed, when the plasma temperature is large the width broadening induced by the potential does not matter much as far as moments are concerned. In the example with magnetospheric plasma conditions (Fig. 5), the measured temperature $(100 \mathrm{eV})$ is much larger than the maximum potential value $(10 \mathrm{eV})$ and correspondingly the estimation ratios do not vary much (less than $6 \%$ for the density and temperature ones, whereas the density goes from $\sim 3 \%$ underestimation to $8 \%$ overestimation). To a lesser extent, these conclusions are also true for the magnetosheath conditions: the temperature remains overestimated by $\sim 25 \%$, whereas the velocity 


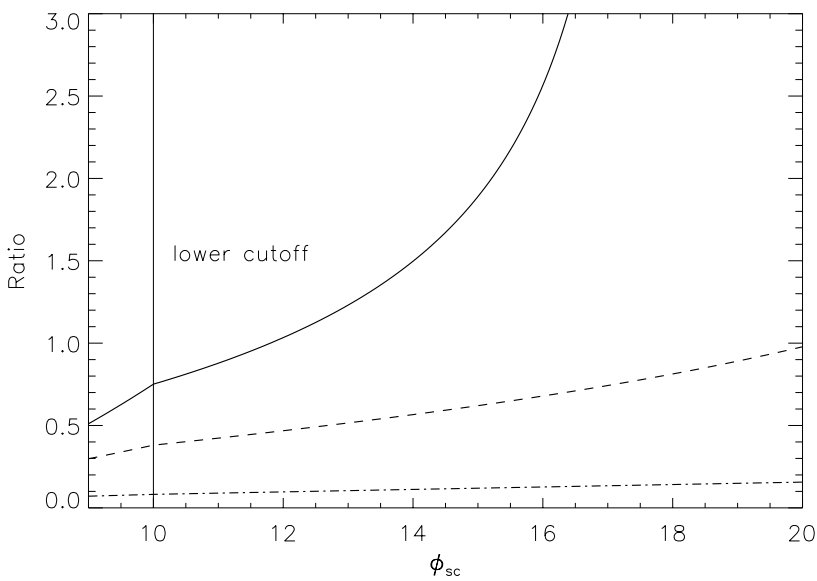

Fig. 6. Minimal density overestimation $r_{N}$ for potentials higher than the detector cutoff (taken here as $10 \mathrm{eV}$ ), i.e. $\sqrt{-\mathcal{E}}>v_{l}$, in the solar wind case (solid line), the magnetosheath case (dashed line) and the magnetosphere case (dot-dashed line).

overestimation varies from 30 to a few percent. The density measure is still most affected by the spacecraft potential and varies from a $25 \%$ underestimation (for zero potential) to a $37 \%$ overestimation.

The variations of $I_{e}$, the estimation index, agree with the above conclusions. For solar wind conditions, this index is close to 1 , indicating that the potential has a great effect on the measure $\left(I_{e}=1\right.$ corresponds, for instance, to an under/over-estimation of $60 \%$ on the three moments). This value is much less in the magnetosheath and magnetosphere. Also, the minimum of this index occurs for potentials close to the critical potential (for which $N_{0}=N$ ) which typically lies in the range $2-6 \mathrm{~V}$, the higher value corresponding to the solar wind. This range agrees well with the capabilities of existing active potential control devices as employed, for example, on Polar (PSI experiment, Moore et al. (1995), with a bias potential $\sim 2 \mathrm{~V}$ ) and Cluster (ASPOC experiment, Torkar et al., 2001, with a bias potential 3-7 V). Such devices basically emit a positive ion beam to counter the positive charge and manage to dynamically stabilize the potential to values close to the bias value.

What happens when the spacecraft potential reaches values higher than the lower energy cutoff? This situation, as far as distribution functions are concerned, is illustrated on the lower panel of Fig. 2. Photoelectrons can now freely enter the detector. However, these particles are not taken into account in our model. The algorithm still works but in the range $v_{l}<v<\sqrt{-\mathcal{E}}$ we set $f=0$, suppressing the photoelectrons which we do not model. This explains the kinks in the variations of the different ratios occuring at the location $\sqrt{-\mathcal{E}}=v_{l}$, as shown in Fig. 6. Therefore, for $\sqrt{-\mathcal{E}}>v_{l}$, in the case of the density, for instance, our method does not give the proper overestimation in the measure, but rather the minimal overestimation, as in a real case, photoelectrons would further add to the density value. We discussed in Sect. 2 how photoelectrons might generally be modeled. Thus, in Fig. 6 we display the minimal density overestimation for different plasma con-

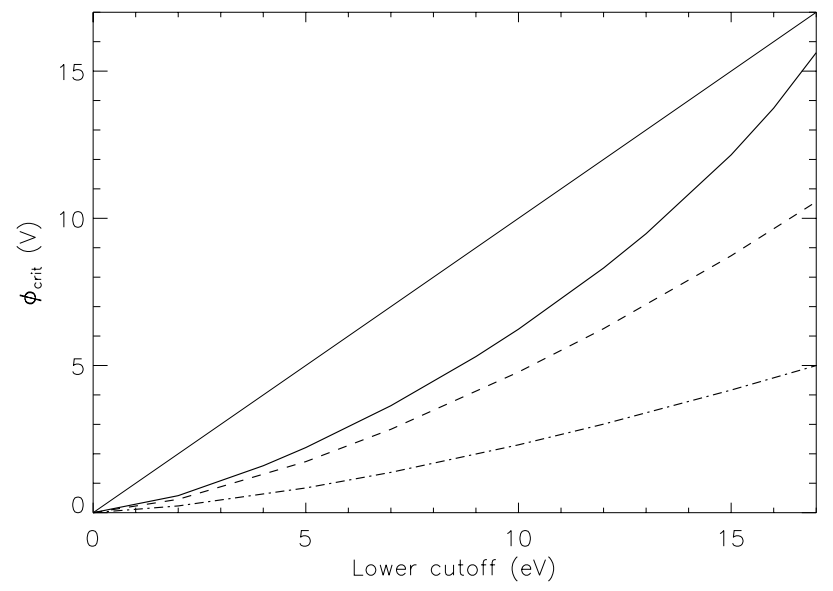

Fig. 7. Critical potential as a function of the lower cutoff of the detector, in the solar wind case (solid line), the magnetosheath case (dashed line) and the magnetosphere case (dot-dashed line).

ditions. For larger potentials (such that $\sqrt{-\mathcal{E}}>v_{l}$ ), the trends, previously described for different plasma regimes, are still present. For instance, $\Phi_{s c}=20 \mathrm{~V}$ (and $m_{e} v_{l}^{2} / 2=10 \mathrm{eV}$ ) leads to a $15 \%$ minimal overestimation for magnetospheric conditions, emphasizing the low dependence of the moments on the spacecraft potential in this region, whereas in the magnetosheath case this value reaches $100 \%$. In the solar wind, the overestimation quickly reaches high values (more than $300 \%$ ). However, in the low density environment, such as the solar wind, the spacecraft potential rarely attains values above $10 \mathrm{eV}$ used as the lower energy cutoff in Fig. 6.

For zero potential, the overestimation (usually occurring for the velocity and temperature measurements) is a 3-D effect. Indeed, in 1-D, when there is no integration over the angular part, the integrand for all moments is strictly positive, and the truncation due to the low energy cutoff can only lead to underestimation. In 3-D the integrand for the moments may be negative at low velocities (see Eq. 12, for instance); the truncation then misses this part and gives a larger result than the total integration $\left(v_{l}=0, v_{u}=\infty\right)$. Trying to sketch 1-D plots for this problem may thus be misleading. The effect of the potential increase is to bring more of the negative integrand area into the sampling (or integration) range. This explains why the velocity is less overestimated at larger potential. As the temperature is the combination of three different pressure terms divided by the density, it is more difficult to discriminate exactly how the (usually small) overestimation occurs.

In Fig. 7 we no longer keep the lower cutoff constant to study its effect on the moment estimation, and more specifically, on the critical potential $\Phi_{\text {crit }}$ previously defined. This quantity is a relevant parameter which bounds the density under/over-estimation regions. Therefore, for a given lower cutoff, when the spacecraft potential is smaller (larger) than $\Phi_{\text {crit }}$ the density will be under(over)estimated. In a region like the magnetosphere where the electron temperature is large, $\Phi_{\text {crit }}$ increases slowly with the lower cutoff, whereas 
in the solar wind the variations are large. Indeed, an increase of the lower cutoff involves smaller relative undersampling for a broad distribution than for a peaked one. Therefore, one needs a smaller increase of the potential to shift the distribution in energy and obtain equality between the measured and real density. Small variations of the lower cutoff have a strong implication on the density estimation, at least in the solar wind where the spacecraft potential rarely exceeds $10 \mathrm{~V}$ : for a cutoff value of $10 \mathrm{eV}$ both under and overestimation can be expected, whereas for a value of $15 \mathrm{eV}$ only underestimation is possible as $\Phi_{\text {crit }} \simeq 12 \mathrm{~V}$. In the solar wind case $e \Phi_{\text {crit }}$ quickly reaches values larger than the lower cutoff which is a domain where our model is not complete (photoelectrons are lacking).

\section{Conclusions}

Revisiting the "perfect" detector concept, we expressed analytically the "measured" moments (density, velocity and temperature) as functions of the (a priori unknown) moments of a Maxwellian distribution function in free space, the spacecraft potential, and lower and upper energy cutoffs of the detector. We numerically inverted the complex nonlinear system derived from the previous equations, and finally obtained the "real" moments. This enabled us to estimate what is the influence of the spacecraft potential by comparing real and instrumentally determined measured moments. Let us summarize the basic findings of the present paper.

- The corrections due to the potential can be very important, especially in the solar wind and magnetosheath environments, by comparison with the case $\Phi_{s c}=0 \mathrm{~V}$ (Song et al., 1997).

- The model shows that the active control of the spacecraft potential to values less than $10 \mathrm{~V}$ minimizes to some extent the discrepancies between real and measured moments. This is due to the offsetting effects of the low energy instrumental cutoff and the residual potential.

- The value of the lower cutoff has significant influence on the estimation of the density, as shown by the variations of the critical potential $\Phi_{\text {crit }}$.

Several lines could be followed to improve the existing model:

- The model of the detector geometry could be improved in the method (see Scime et al., 1994, for instance), although this would destroy the angular symmetry which permitted several simplifying analytical steps.

- For $e \Phi_{s c}>m_{e} v_{l}^{2} / 2$, the contribution of the photoelectrons could be added. Such a situation happens when the plasma density is very low (the lobes, for instance). This requires a correct model of the photoelectron distribution to be implemented (see, for instance, Grard, 1973; Pedersen, 1995).
- A potential barrier outside the spacecraft (a few spacecraft radii away) exists when the photoelectrons dominate the space charge around it. This barrier may affect the plasma measurements in an equivalent way as raising the lower energy cutoff. Both plasma and photoelectron distributions are then modified. However, this non-monotonic potential behaviour is difficult to determine (see, for instance, Whipple, 1976; Thiébault et al., 2004).

- As the method is partly numerical, it would be possible to assume distribution functions other than Maxwellian. Anisotropies related to the magnetic field direction would again break the angular symmetry and increase the numerical complexity.

However, any improvement will not change the basic feature of the method: it deals with pre-computed moments which may be affected by calibration deficiency (energy efficiency, geometry factor, etc.). In the complex process of correcting moments from spacecraft potential and energy cutoffs, it may then be more valuable to use data from a lower level, namely to work directly with the distribution functions. For instance, it is possible to apply a potential correction directly to the 3-D distribution functions by shifting the energy channels of the corresponding energy, and then recompute the moments from these new distributions; any holes left in the distributions may be filled by some simple model fit. If this seems the most obvious solution, it often does not offer a good alternative as the 3-D products from an energy spectrometer cannot always be transmitted with high time resolution to the ground (they require too much telemetry). The result is then, at best, moments with degraded time resolution. A fix to this problem could be to perform a similar method using the pitch-angle distributions which are generally transmitted at a higher rate. However, this is done at the expense of invoking an assumption of gyrotropy. In conclusion, no perfect method to correct spacecraft potential effects has been designed yet, and the one presented in this paper, with its limitations but with also its simplicity, can provide a crude and quick improvement from the measured moments to their underlying true values.

Acknowledgements. V. Génot is supported by a UK PPARC grant.

Topical Editor T. Pulkkinen thanks G. Paschmann for his help in evaluating this paper.

\section{References}

Bouhram, M., Dubouloz, N., Hamelin, M., Grigoriev, S. A., Malingre, M., Torkar, K., Veselov, M. V., Galperin, Y., Hanasz, J., Perraut, S., Schreiber, R., and Zinin, L. V.: Electrostatic interaction between Interball-2 and the ambient plasma, 1. Determination of the spacecraft potential from current calculations Ann. Geophys., 20, 365, 2002.

Décréau, P. M. E., Fergeau, P., Krannosels'kikh, V., Lévêque, M., Martin, Ph., Randriamboarison, O., Sené, F. X., Trotignon, J. G., Canu, P., and Mögensen, P. B.: Whisper, a Resonance Sounder 
and Wave Analyser: Performances and Perspectives for the Cluster Mission, Space Sci. Rev., 79, 157, 1997.

Forest, J., Eliasson, L., and Hilgers, A.: A New Spacecraft Plasma Simulation Software, PicUp3D/Spis, 7th Spacecraft Charging and Technology Conference, p.515-520, ESA/SP-476, ESAESTEC, Noordwijk, The Netherlands, 23-27 April 2001.

Grard, R. J. L: Properties of the satellite photoelectron sheath derived from photoemission laboratory measurements, J. Geophys. Res., 78, 2885, 1973.

Hilgers, A., Holback, B., Holmgren, G., and Bostrom, R.: Probe measurements of low plasma densities with applications to the auroral acceleration region and auroral kilometric radiation sources, J. Geophys. Res., 97, 8631, 1992.

Johnstone, A. D., Alsop, C., Burge, S., Carter, P. J., Coates, A. J., Coker, A. J., Fazakerley, A. N., Grande, M., Gowen, R. A., Gurgiolo, C., Hancock, B. K., Narheim, B., Preece, A., Sheather, P. H., Winningham, J. D., and Woodliffe, R. D.: Peace: a Plasma Electron and Current Experiment, Space Sci. Rev., 79, 351, 1997.

Laakso, H. and Pedersen, A.: Ambient electron density derived from differential potential measurements, Measurement Techniques in Space Plasmas, edited by Borovsky, J., Pfaff, R., and Young, D., AGU Monograph 102, 49, AGU, Washington DC, 1998.

Lin, R. P., Anderson, K. A., Ashford, S., Carlson, C., Curtis, D., Ergun, R., Larson, D., McFadden, J., McCarthy, M., Parks, G. K., Rème, H., Bosqued, J. M., Coutelier, J., Cotin, F., D’Uston, C., Wenzel, K.-P., Sanderson, T. R., Henrion, J., Ronnet, J. C., and Paschmann, G.: A Three-Dimensional Plasma and Energetic Particle Investigation for the Wind Spacecraft, Space Sci. Rev., $71,125,1995$.

Meyer-Vernet, N., Hoang, S., Issautier, K., Maksimovic, M., Manning, R., Moncuquet, M., and Stone, R. G.: Measuring plasma parameters with thermal noise spectroscopy, Measurement Techniques in Space Plasmas, edited by Borovsky, J., Pfaff, R., and Young, D., AGU Monograph 102, 205, AGU, Washington DC, 1998.

Moore, T. E., Chappell, C. R., Chandler, M. O., Fields, S. A., Pollock, C. J., Reasoner, D. L., Young, D. T., Burch, J. L., Eaker, N., Waite Jr., J. H., McComas, D. J., Nordholt, J. E., Thomsen, M. F., Berthelier, J. J., and Robson, R.: The Thermal Ion Dynamics Experiment and Plasma Source Instrument, Space Sci. Rev., 71, 409, 1995.
Paschmann G., Fazakerley, A. N., and Schwartz, S. J.: Moments of plasma velocity distributions, Analysis Methods for MultiSpacecraft Data, Chapter 6, edited by Paschmann, G. and Daly, P. W., ISSI scientific report, 1998.

Pedersen, A.: Solar wind and magnetosphere plasma diagnostics by spacecraft electrostatic potential measurements, Ann. Geophys., 13, 118, 1995.

Press, W. H., Teukolsky, S. A., Vetterling, W. T., and Flannery, B. P.: Numerical Recipes in Fortran 77: The Art of Scientific Computing, Cambridge University Press, 1992.

Salem, C., Bosqued, J.-M., Larson, D. E., Mangeney, A., Maksimovic, M., Perche, C. , Lin, R. P., and Bougeret, J.-L.: Determination of accurate solar wind electron parameters using particle detectors and radio wave receivers, J. Geophys. Res., 106, 10, $21701,2001$.

Scime, E. E., Phillips, J. L., and Barne, S. J.: Effects of spacecraft potential on three-dimensional electron measurements in the solar wind, J. Geophys. Res., 99, 8, 14 769, 1994.

Singh, N., Leung, W. C., Moore, T. E., and Craven, P. D.: Numerical model of the plasma sheath generated by the plasma source instrument aboard the Polar satellite, J. Geophys. Res., 106, 9, $19179,2001$.

Song, P., Zhang, X. X., and Pashmann, G.: Uncertainties in plasma measurements: effect of lower cutoff energy and spacecraft charge, Planet. Space Sci., 45, 2, 255, 1997.

Szita, S., Fazakerley, A. N., Carter, P. J., James, A. M., Trávníček, P., Watson, G., André, M., Eriksson, A., and Torkar, K.: Cluster PEACE observations of electrons of spacecraft origin, Ann. Geophys., 19, 1, 2001.

Thiébault, B., Hilgers, A., Sasot, E., Laakso, H., Escoubet, P., Génot, V., and Forest, J.: Potential barrier in the electrostatic sheath around a magnetospheric spacecraft, J. Geophys. Res., accepted, 2004.

Torkar, K., Riedler, W., Escoubet, C. P., Fehringer, M., Schmidt, R., Grard, R. J. L., Arends, H., F. Rüdenauer, Steiger, W., Narheim, B. T., Svenes, K., Torbert, R., André, M., Fazakerley, A., Goldstein, R., Olsen, R. C., Pedersen, A., Whipple, E., and Zhao, H.: Active spacecraft potential control for Cluster - implementation and first results, Ann. Geophys., 19, 1289, 2001.

Whipple Jr., E. C.: Theory of the spherically symmetric photoelectron sheath - A thick sheath approximation and comparison with the ATS 6 observation of a potential barrier, J. Geophys. Res., $81,4,601,1976$. 